\title{
Study on Intelligent Alarm System for Gas Concentration Detection
}

\author{
JuanHe,ShuaiKANG,Guang-yi LUO \\ Department of physics and mechanical-electrical engineering, Zunyi Normal College
}

Keywords: Gas concentration; Digital technology; Alarm system

Abstract: Aiming at the coal mine safety production, a kind of intelligent alarm system has been designed based on DSP technology, each part has been introducteddetailly and the system is simulated by CCS software. The simulation results show the system is correctness and feasibility, which proves that the system has certain application value in coal mine safety production.

\section{Introduction}

Mine gas is a mixture of gases withCH4, carbon dioxide, nitrogen, hydrocarbons and trace rare gases. The $\mathrm{CH} 4$ is the main component of Mine gas, and the ignition temperature is very low, when the content of air reaches 5\% 15\% and encounters fire, the gas will explode, and which is colorless and odorless. The leakage gas is not easy to be found, and which threats to the safety production of coal mine and underground work personnel seriously, so it is necessary to early detecte and early warn of theMine gas in the mine environment. And gas accumulation may cause gas accident, it is a very important work to control the coal mine gas dynamics in time. Therefore, it has become one of the most urgent tasks for the safety of coal mine safety [1-2] production monitoring by strengthening the gas management and improving the monitoring level of gas detection. Gas concentration detection and alarm device is an effective tool to monitor the dynamic of the gas in the mine. In this paper, the DSP control is used to study the intelligent alarm system of gas concentration detection[3-6].

\section{Overall System Structure}

Coal mine gas concentration detection alarm systembased on DSP is mainly composed of DSP TMS320LF2407A, which includes the power supply circuit, gas concentration detection alarm device, signal amplification circuit, A/D converter circuit, LED display circuit, luminous alarm circuit, voice alarm circuit. The system diagram of the coal mine gas concentration detection and alarm system based on DSP is shown in Figure 1.

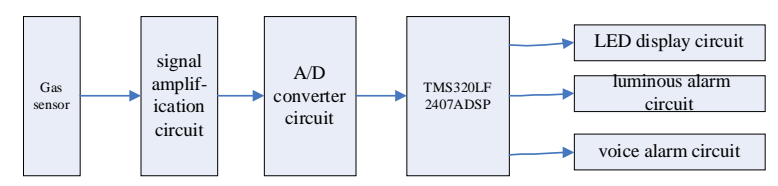

Fig.1Thesystem diagram of the coal mine gas concentration detection and alarm system based on DSP

The gas concentration information from detecting will be transmitted to the DSP chip through the gas concentration detection alarm device, after processing with DSP chip, information of gas concentration will be display in the LED display device and also if the gas concentration exceeds the set value, the coal mine gas concentration detection alarm device will immediately send out sound and light alarm and remind coal mine production personnel to take appropriate safety 
measures timely.

\section{Gas Concentration Detection Circuit}

There are many methods to detect gas concentration, such as thermal conductivity method, optical interference method, infrared spectrum absorption method, ultrasonic method, semiconductor oxide gas sensor method and carrier catalytic gas sensor detection method. The most commonly method is carrier catalytic gas sensormethod, which has high sensitivity, wide measurement range, small size and low price, this method is widely used in the coal mine of our country. The method uses the resistance change to determine the gas concentration through generating heat from the oxidation by methane in the catalytic element. The Figure 2 is gas concentration detection circuit, the R3 and the $\mathrm{R} 4$ are expressed in black element and whiteelement, $\mathrm{J} 1$ is connected with the input signal of the amplifier. We Set catalytic element R3 as a working element and R4 as a compensation element.The measuring bridge circuit is in equilibriumwithout gas, the same constant current flows $\mathrm{R} 3$ and R4.whenthere is coal gas, gas and oxygen react on the surface of the components so as to increase the resistance of the element, the bridge will lose balance, which produces the positive output voltage signal with gas concentration, and We will get the level of gas concentration by measuring the size of this signal.

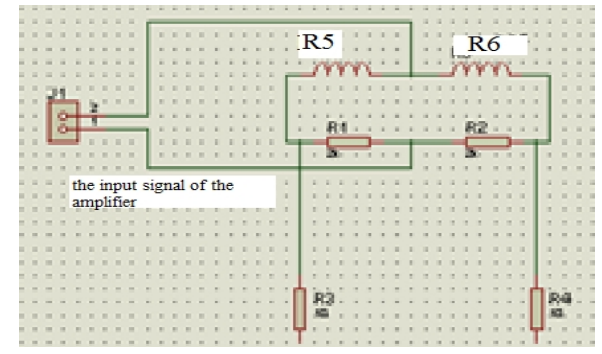

Fig.2 gas concentration detection circuit

\section{Gas Signal Processing Circuit}

The gas signal processing circuit designed in this paper consists of two parts: Part one is analog signal amplifying circuit and the other is analog- digital conversion circuit.

In analog signal amplifying circuit, INA122 is chosen as the main component of the signal amplifying circuit. INA122 is a precision instrument amplifier with America Burr-Brown company recently. There are extremely low quiescent current and a wide power supply range, with very low input offset, input drift and excellent common mode suppression ratio through the combination of two operational amplifier, the gain can be sets out 5 - 10000 times the magnifying power through an external resistor, which has been widely used in data acquisition system. Analog signal amplifier circuit is shown in Figure 3.

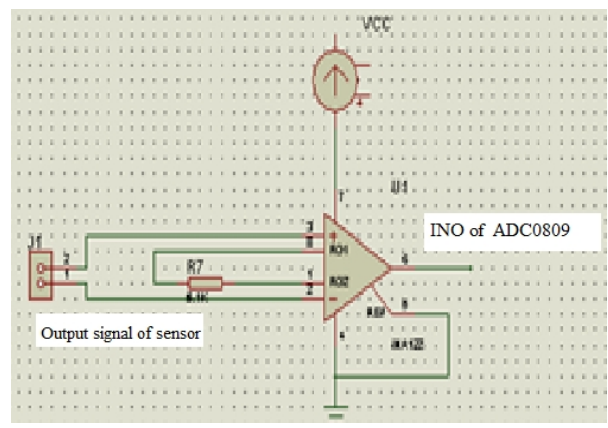

Fig 3 Analog signal amplifier circuit 
In the measurement of $0 \% \sim 5 \% \mathrm{CH} 4$, the output voltage signal range of the detection circuit is $0 \mathrm{~V}$ $\sim 1125 \mathrm{mV}$. The range of output voltage signal of the alarm device is amplified to $0 \sim 5 \mathrm{~V}$, and the gain of INA122 is obtained in the circuit design.

$$
\mathrm{G}=\frac{5 \mathrm{~V}}{112.5 \mathrm{mV}} \approx 44
$$

The resistance value of the resistor RG (i.e. R7 in Figure 3) is:

$$
\mathrm{R}_{\mathrm{G}}=\frac{200}{\mathrm{G}-5} \approx 5.1 \mathrm{~K} \Omega
$$

In the digital analog conversion circuit, the ADC0809 chip is chosen as the main device of the analog to digital conversion circuit. ADC0809 is a 8 bit successive approximation of the A/D converter, which can be divided into 8 analog signal acquisition and A/D conversion, the output digital signal can be directly connected with the data bus of single chip microcomputerthrough the three state buffer.

\section{DSP System Design}

\section{(1) DSP select}

DSP TMS320LF2407A chip is used for Hardware system. TMS320LF2407A has a lot of peripheral resources, which provides the two event management module of EVA and EVB, two 16 bit global counter, eight adjustable pulse width modulation channel PWM, three external event timing of sample acquisition unit synchronization of 16 channel high performance 10bit ADC, asynchronous serial communication interface (SCI), synchronous serial peripheral interface (SPI), CAN bus 2.0 interface and the conversion rate is $500 \mathrm{~ns}$.

(2) keyboard circuit

The required keys for Coal mine gas concentration detection alarm system is less, wedon't use the expansion of the chip and directly use the DSP idle port to design and implement the keyboard. The design of the keyboard circuit is shown in Figure 4.There are 40 independent programmable I / $\mathrm{O}$ port in DSP chip, we can match four ports for detecting the four key input and four buttons is shown respectively in Figure 4, "detection and alarm operation" key is used to start mine gas concentration detection alarm device operation, "detection and alarm stop" button is to stop coal mine gas concentration detection alarm device operation, "light alarm detection" button for the detection of coal mine gas concentration detection alarm device luminous alarm is able to normal operation, "voice alarm detection "button for detection of coal mine gas concentration detection alarm device alarm vocalizations is able to normal operation.

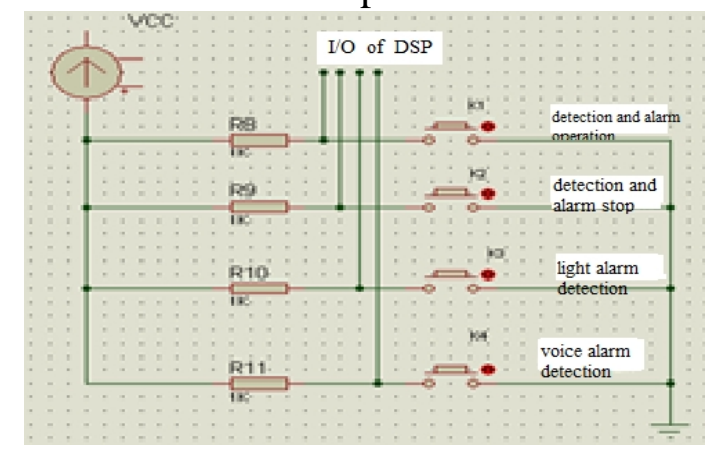

Fig.4 the keyboard circuit

(3) display circuit

In the display circuit, the range of gas concentration detection alarm device is $0 \% \sim 5 \%$, and the 4 LCD display is designed to display the concentration of gas. The display format is "X.XXX", which indicates that the concentration of the measured gas is "X.XXX\%". 
(4) alarm circuit

When the gas concentration from the gas sensor to measure is over the range of alarm, the alarm circuit is to start the action and send out sound and light alarm information. The sound and light alarm circuit is shown in Figure 5. When the gas concentration through the gas sensor to measure is over $1 \%$, the gas concentration exceeds the light emission warning light, while the gas concentration exceeds the emission alarm speaker sound, at this time the remote monitoring personnel will receive the information, immediately alert the coal mine production personnel to take the appropriate security measures.

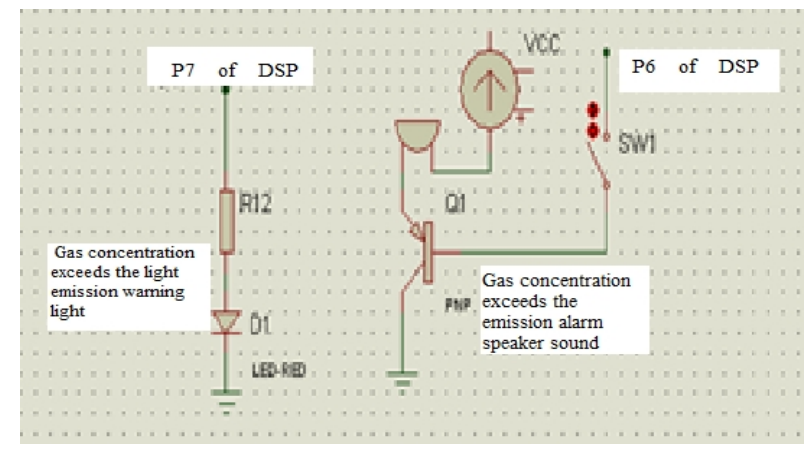

Fig.5 The sound and light alarm circuit

Main function of coalmine gas concentration detection alarm system based on DSP includes four parts as follows: (1)initialization of DSP system, the working parameters of the definition of each peripheral function chips, (2) gas concentration detection alarm device to detect the concentration of gas and amplified transmission to the A / D converter, (3) to start A/D converter, The A/D conversion result is transmitted to the DSP, (4) results of A/D conversion will be processed by DSP, one is sent to LCD display shows the, the second is to judge whether gas concentration is exceeded, if gas concentrationsis excessive, alarm circuit will be start and send sound and light alarm information.

\section{Simulation}

We use CCS3.3 software to design the simulation model of the intelligent alarm system for gas concentration detection based on DSP. In CCS software, there is no gas sensor model, we use the the output voltage signal ofpotentiometer to simulate the output voltage signal of gas sensor in the simulation. The output voltage signal of the potentiometer is adjusted respectively to simulate the output voltage signal of the gas sensor, and the analog voltage signal is converted bythe ADC0808, which is converted to the DSP, and the signal is processed by DSP after the conversion. When the gas concentration is lower than $1 \%$, the normal working light (green) will be lighted and indicating that the current gas concentration is not exceeded. When the gas concentration is higher than $1 \%$, but less than $1.5 \%$, the general exceeding alarm indicator light (yellow) will be lighted and indicating that the current gas concentration exceeds the minimum value. When the gas concentration is higher than $1.5 \%$, the serious warning lights (red) will be lighted and indicating that the current gas concentration exceeds the maximum limit.

\section{Conclusions}

The gas detection and alarm system based on DSP digital technology is mainly made of by the TMS320LF2407A DSP, power supply circuit, gas concentration detection alarm device, a signal amplifying circuit, A/D conversion circuit, LED display circuit, a light alarm circuit and a sound 
alarm circuit part and every part were introduced in detail, at the same time, the simulation has been done with the CCS software in the laboratory, which verifies the correctness and feasibility of the system. It is proved that this system has a certain practical value in gas concentration detection and has a strong theoretical basis for the safety production of the coal mine.

\section{ACKNOWLEDGMENTS}

This work is supported by the Science and Technology Foundation of Guizhou Province under Grant (No.J [2013]2306, No. J [2012]2345, No.JLKZS[2014]05 and No.JLKZS[2015]7043)and the Special Foundation of Governor of Guizhou Province for Science and Technology and Education Talents Grant (No. [2012]87) and the Key Project of Education Department of Guizhou province Grant( No [KY(2013)171]), This work is also supported by The Education Department of Guizhou Province electronic manufacturing production base (No. [2014]230) and the key discipline of Guizhou province (No.[2013]18).

\section{REFERENCE}

[1] XuePengqian, Pan Yumin, Zhang Tao, et al. Coal mine safety detection technology and monitoring system [M]. Beijing: China Coal Industry Press, 2010

[2] Zhang Congli, Zhang Hexiang. the applicationof information fusion technology in coal mine safety prediction . computer simulation for [J]..2012 (5): 201 205.

[3] Liu Lina, Liu Renqing. Current situation and comparative study of gas detection equipment[J]. Modern electronic technology, 2011, 34 (15): 159-160.

[4] Bai Yan,TengChunyang, Wei Yuchen, et al. Design of gas concentration detector[J]. China Science and Technology Expo, 2010 (26): 45

[5] Gao Yunfeng. highYunfeng. Multi point distributed coal mine gas monitoring system based on DSP [J].Coal technology.2011 (2):113 115.

[6]Wang Zhenghong, Ma Yiwu, Zhang Zaochun. Real time monitoring system of gas concentration based on C8051F040 and sensor array [J].Instrument technique and sensor, 2007 (8): 26 29. 